\title{
Renal Function in Acromegaly - Experience from a Portuguese Centre
}

Carlos Tavares Bello ; Fonseca, RC; Sequeira Duarte, J ; Vasconcelos,C Hospital de Egas Moniz - Centro Hospitalar de Lisboa Ocidental
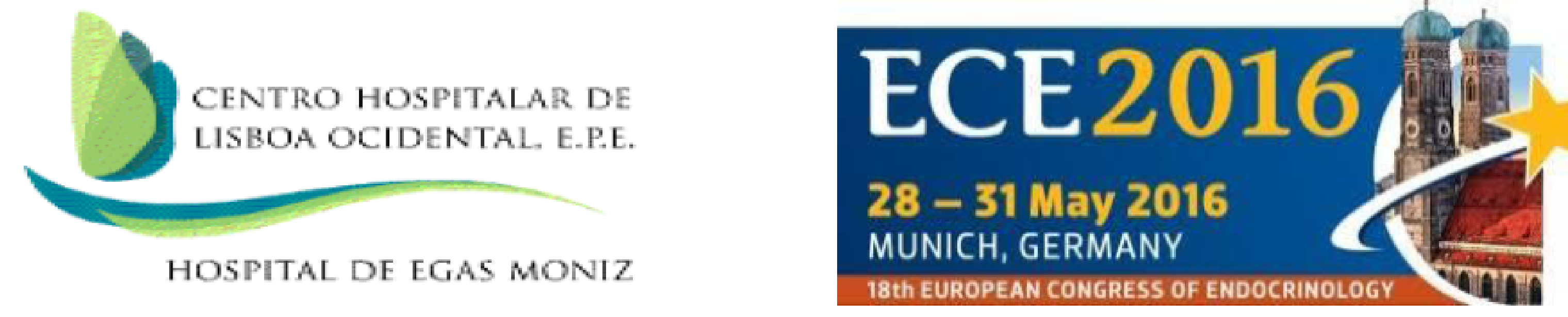

\section{INTRODUCTION}

The Nephron is a known target organ of the Growth Hormone/Insulin-like factor-1 axis.

They influence glomerular and tubular function having an important physiological role in water and electrolyte balance, especially in Acromegaly. The aim of the study was to investigate renal function in acromegalic patients during active disease and remission and evaluate hormonal impact on renal function markers.

\section{METHODS}

A retrospective, longitudinal, observational analysis was performed.

Variables analyzed: Creatinine, glomerular filtration rate (GFR), IGF1/GH and the remainder pituitary hormones.

Descriptive statistical methods ; Pearson correlations and student T-test were used to analyze numerical data.

Results are presented as mean and standard deviation.

\section{RESULTS}

- 52 Acromegalic patients.

- Females: 69.2\%

- Age $55,5 \pm 14,8$ years.

- The underlying etiology was a pituitary adenoma in $100 \%$.

- Surgery was undertaken by $82,6 \%$

- Medical therapy was used in $92,2 \%$

- $19.2 \%$ were treated with Radiotherapy.

- Disease control - $71.2 \%$.

\begin{tabular}{|c|c|}
\hline Variable & \\
\hline Creatinine $(\mathrm{mg} / \mathrm{dL})$ & $0,84 \pm 0,21$ \\
\hline GFR (CKD-EPI) $\mathrm{mL} / \mathrm{min} / 1,73 \mathrm{~m} 2$ & $86 \pm 22,9$ \\
\hline GH $(\mathrm{ng} / \mathrm{mL})$ & $2,7 \pm 5,4$ \\
\hline IGF1 $(\mathrm{ng} / \mathrm{mL})$ & $288,3 \pm 217$ \\
\hline
\end{tabular}

\begin{tabular}{|c|c|}
\hline Variable & \\
\hline Age & $55,5 \pm 14,8$ \\
\hline Male / Female & $30,8 / 69,2$ \\
\hline Microadenoma & $21,2 \%$ \\
\hline Macroadenoma & $76,9 \%$ \\
\hline Invasive Adenoma & $1,9 \%$ \\
\hline Surgery as initial therapy & $82,6 \%$ \\
\hline Pharmacological therapy as initial treatment & $17,3 \%$ \\
\hline
\end{tabular}

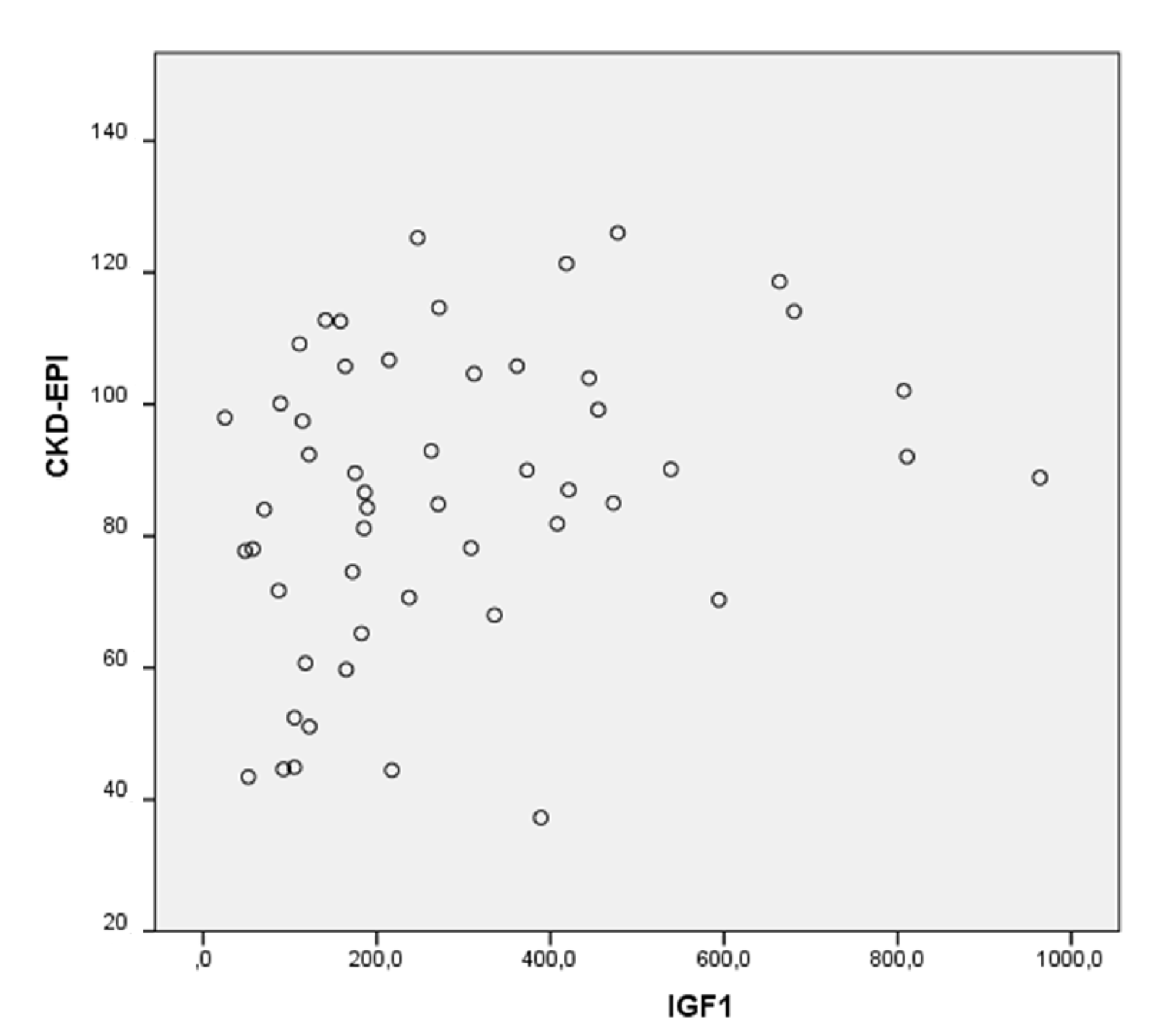

\begin{tabular}{ccccc} 
P values & Creatinine & CKD-EPI & GH & IGF-1 \\
\hline Creatinine $(\mathrm{mg} / \mathrm{dL})$ & & $<0,001$ & 0,09 & 0,08 \\
CKD-EPI & $<0,001$ & & 0,051 & $\mathbf{0 , 0 2 5}$ \\
GH & 0,09 & 0,051 & & $<0,001$ \\
IGF-1 & 0,08 & $\mathbf{0 , 0 2 5}$ & $<0,001$ & \\
\hline
\end{tabular}

- Statistical significant correlations were found between IGF-1 and eGFR $(p 0,025)$.

- Patients in remission had a lower mean eGFR (79 vs $89 \mathrm{~mL} / \mathrm{min} / 1.73 \mathrm{~m} 2)$.

- Degree of elevation of IGF1 from the upper normal range (expressed in \%) was correlated with eGFR, creatinine and metabolic parameters (Fasting blood glucose and glycated hemoglobin) with statistical significance.

\section{CONCLUSIONS}

The current study demonstrates consistently a possible positive correlation between IGF1/GH axis and renal function markers. Despite being a retrospective study, the sample size may further support the relevance of the GH/IGF1-renal axis in Acromegaly. 